\title{
Development and Evaluation of High Oxaliplatin Loaded CS-g-PNIPAAm Co-Polymeric Nanoparticles for Thermo and pH Responsive Delivery
}

\author{
Archana Sidagouda Patil ${ }^{\star}$, Anand Panchakshari Gadad \\ Department of Pharmaceutics, KLE University College of Pharmacy Belagavi, KLE Academy of Higher Education and Research \\ Belagavi-590010, Karnataka, INDIA.
}

\begin{abstract}
Background: Chitosan-g-poly(N-isopropylacrylamide) (CS-g-PNIPAAm) co-polymer was reported as a much efficient drug carrier but it shows very low percentage of drug loading in to the nanoparticles. Objective: The objective of the present study was to develop highly loaded $\mathrm{pH}$ and thermo responsive CS-g-PNIPAAm co-polymeric nanoparticles for tumor specific oxaliplatin delivery. Methods: CS-g-PNIPAAm co-polymer was synthesized by surfactant free dispersion copolymerization method and characterized for its structure (FTIR1H NMR), morphology and lower critical solution temperature (LCST). Different drug loading approaches like direct drug loading during polymerization and self-assembly methods were used. In direct drug loading method the chitosan concentration was varied and in self assembly method the polymer ratio and sonication time were varied for study. Drug loaded nanoparticles were evaluated for particle size, zeta potential, percent drug loading efficiency, percent drug content and in-vitro drug release study. Results: It was observed that, self-assembly method give a high amount of oxaliplatin loaded nanoparticles. Further, in direct loading method, as the concentration of chitosan in co-polymer increases, the percent drug loading and drug release at above LCST of copolymer also increases. Nanoparticles prepared by self assembly method (F-5) showed the maximum drug release at above LCST $(\mathrm{pH} \mathrm{7.2)} \mathrm{and} \mathrm{trace} \mathrm{amount} \mathrm{of} \mathrm{drug} \mathrm{release} \mathrm{below}$ LCST ( $\mathrm{pH} 7.5$ ), indicating thermo and $\mathrm{pH}$ responsive delivery of oxaliplatin. Conclusion: In conclusion, higher oxaliplatin loading can be achieved by using self-assembly method with sonication time $5 \mathrm{~min}$ and drug:polymer ratio of about 3:10 and oxaliplatin release can be controlled by varying chitosan concentration in co-polymer.
\end{abstract}

Key words: Oxaliplatin, Chitosan-g-poly( $\mathrm{N}$-isopropylacrylamide), Self assembly method, Thermo and $\mathrm{pH}$ responsive delivery.

\section{INTRODUCTION}

Environment-sensitive or intelligent polymers are those which show sharp and reversible transition in physical/chemical properties in response to small changes in environmental conditions. ${ }^{1}$ The response of polymer system is modulated by different stimuli which can be classified as internal stimulus (e.g. pH, glucose, redox potential and lysosomal enzymes) and external stimulus (e.g. temperature, magnetic field, ultrasound and light). ${ }^{2}$ Dual stimuli responsive polymers which respond to the combination of two signals such as $\mathrm{pH} /$ temperature, $\mathrm{pH} /$ redox, $\mathrm{pH}$ / magnetic field have been widely investigated in the areas of controlled drug delivery, ${ }^{3-5}$ diagnostics, ${ }^{6,7}$ sensors, ${ }^{8,9}$ chromatographic separations $^{10,11}$ and tissue engineering. ${ }^{12,13}$ Temperature and $\mathrm{pH}$ are typical variable parameters in any biological system, which has prompted the use of temperature and $\mathrm{pH}$ dual responsive polymers in intelligent drug delivery. In the case of solid tumors, extracellular $\mathrm{pH}(6-7)$ is lower than that of surrounding tissues and blood (7.4) as well as, the temperature around tumor cells is always higher than that of nor-
Submission Date: 18-03-2017; Revision Date: 15-06-2017; Accepted Date: 25-09-2017

DOI: 10.5530/ijper.52.1.7 Correspondence:

Mrs. Archana Sidagouda Patil,

Assistant Professor, Dept. of Pharmaceutics, K.L.E.U's College of Pharmacy, Belagavi, Karnataka, INDIA. Phone no: +91-9916883344; E-mail: archupharma@gmail. com

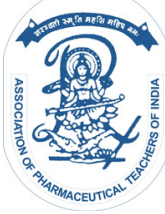

www.ijper.org 
mal body temperature. ${ }^{14,15}$ Taking these differences as a basic concept, various $\mathrm{pH}$ and temperature responsive polymers were synthesized and utilized as carriers for stimuli-responsive delivery of anti-cancer drugs. ${ }^{16-20}$ Though all these reported polymeric nanoparticles have shown tremendous potential for targeted drug delivery, they have a very low drug loading capacity. Among them, due to the closer LCST $\left(32^{\circ} \mathrm{C}\right)$ of PNIPAAm to normal body temperature, biocompatibility and non-toxicity, chitosan-g-poly(N-isopropylacrylamide) (CS-g-PNIPAAm) co-polymer was most extensively studied as a carrier for anticancer drugs to achieve thermo and $\mathrm{pH}$ responsive delivery of drugs to the intended sites with minimal or no adverse effects in cancer therapy. Chitosan is a natural polysaccharide with high biocompatibility and shows $\mathrm{pH}$-responsive property due to protonation-deprotonation equilibrium of $\mathrm{NH}_{2}$ groups present in its chains. ${ }^{21}$ PNIPAAm is much extensively studied thermo-responsive polymer with LCST of about $32^{\circ} \mathrm{C}$, which is very useful for its biomedical applications. PNIPAAm is water soluble below its LCST and undergo coil-to-globule transition above LCST due to hydrophilic-hydrophobic interactions. $^{22}$

$\mathrm{Li} \mathrm{F}$ and coworkers evaluated camptothecin and paclitaxel loaded poly NIPAAm/chitosan nanoparticles as a $\mathrm{pH}$-sensitive carrier for tumor targeting with encapsulation and loading efficiencies for camptothecin were found to be $73.7 \%$ and $8.4 \%$, respectively and that of paclitaxel were found to be $85.7 \%$ and $9.6 \%$, respectively. ${ }^{23,24}$ Duan $\mathrm{C}$ et al. developed CS-g-PNIPAm co-polymer based $\mathrm{pH}$-responsive nanogels for tumortargeting delivery of oridonin with encapsulation efficiency of $86.3 \%$ and the loading efficiency of $5.34 \%$ only. ${ }^{25}$ Zang $\mathrm{T}$ et al. prepared self-assembled thermo sensitive polyelectrolyte complex (PEC) nanoparticles from chitosan-graft-poly(N-isopropylacrylamide)/carboxymethyl cellulose for entrapment and release of 5-flurouracil. In order to load optimal amount of drug in to nanoparticles, the feed of 5-FU was varied from 5 to $100 \%$ for PEC nanoparticles. The results showed that the drug-loading content increased from $1.67 \%$ to $10.70 \%$, whereas the entrapment efficiency decreased from $34.02 \%$ to $11.98 \%$ with the increase of feed drugs. This was because, when the maximum drug loading was achieved, the excess of drug was dissolved in the solution or adsorbed on the surface of nanoparticles resulting in increased drug content and decreased loading efficiency. ${ }^{26}$ Recently, Huang $\mathrm{C}$ et al. prepared $\mathrm{pH}$ and thermo-sensitive chitosan-PNIPAAm core-shell nanoparticles and evaluated as drug carriers with doxycycline hyclate with encapsulation efficiency and loading content of 60.3 and $3.02 \%$, respectively. ${ }^{27}$ In these studies CS-g-PNIPAAm co-polymer as a carrier has shown expected response to release drug at the target site but unfortunately it was resulted very low percentage of drug loading in to the nanoparticles, which is a major problem for economical production of such delivery systems. To address this issue, in the present study, efforts were exploited to develop a thermo and $\mathrm{pH}$ responsive CS-g-PNIPAAm co-polymeric nanoparticulate delivery system for oxaliplatin and intended to improve its percent drug loading efficiency (LE) and drug content (DC) with controlled drug release by using different drug loading methods and other parameters affecting drug loading. First, the CS-g-PNIPAAm co-polymer was synthesized by surfactant free dispersion copolymerization method, characterized and applied as carrier for oxaliplatin. Drug loaded nanoparticles prepared by varying methods were evaluated for morphology, particle size, zeta potential, loading efficiency, drug content and in-vitro drug release study.

\section{MATERIALS AND METHOD}

\section{Materials}

Chitosan (degree of deacetylation $>90 \%$ ) was obtained as a gift sample from Central Institute of Fisheries Technology, Cochin, India. Oxaliplatin pure drug was obtained as gift sample from Emcure Pharmaceuticals Limited, Pune, India. Ammonium persulphate (APS), $\mathrm{N}, \mathrm{N}$-methylenebisacrylamide (MBA), $\mathrm{N}$-isopropylacrylamide (NIPAAm) and glacial acetic acid were purchased from Acros (Geel, Belgium). Dialysis membrane with molecular weight cutoff of 12000-14000 Da was purchased from HiMedia Laboratories Pvt. Ltd. Mumbai, India (LA 401-HiMedia). India. All the other chemicals used were of analytical grade.

\section{Preparation of chitosan-g-PNIPAAm co-polymeric nanoparticles:}

Chitosan-g-PNIPAAm (CS-g-PNIPAAm) co-polymeric nanoparticles (blank nanoparticle-F-1) were prepared by a surfactant free dispersion co-polymerization method with some modifications. ${ }^{28}$ Chitosan solution was prepared by using $1 \%$ glacial acetic acid with continuous stirring for $24 \mathrm{~h}$ at room temperature. Copolymerization was carried out in nitrogen atmosphere. To the chitosan solution, NIPAAm (1 gm) and MBA $(30 \mathrm{mg})$ were added with vigorous stirring and the temperature was raised to $50^{\circ} \mathrm{C}$ and then, APS (100 $\mathrm{mg}$ ) was added to initiate the polymerization. The reaction medium turned turbid within 10 min and was allowed to proceed for $3 \mathrm{~h}$ at $50-55^{\circ} \mathrm{C}$. The reaction solution was purified by 
transferring to a dialysis bag (LA 401-High media) and dialyzed against 1 liter capacity of water for a week at room temperature. The dialyzing solution was changed on the daily basis. After dialysis, the solution containing homopolymer PNIPAAm and CS-g-PNIPAAm was obtained. The homopolymer PNIPAAm was removed by the methanolic extraction at $20^{\circ} \mathrm{C}$ for $48 \mathrm{~h}$ to get the pure CS-g-PNIPAAm which was then lyophilized at $-99^{\circ} \mathrm{C}$ with 0.01 mbar pressure and total monomer conversion (X \%) was calculated by gravimetric analysis using the following equation.

$$
\mathrm{X} \%=\frac{\left(W_{\text {Dry }}-W_{C S}-W_{\text {APS }}\right)}{\left(W_{\text {NIPAAM }}+W_{\text {MBA }}\right)} \times 100
$$

Where, $\mathbb{W}_{\text {Dry }}$ is the weight of polymers (CS-g-PNIPAAm and PNIPAAm), $W_{C S}, W_{\text {APS }}, W_{\text {NIPAAm }}$ and $W_{\text {MBA }}$ are the weights of chitosan, APS, NIPAAm monomer and MBA in the feed, respectively.

\section{Characterization of chitosan-g-PNIPAAm}

The synthesized co-polymer was characterized by using Fourier-transform infrared (FTIR) and ${ }^{1} \mathrm{H}$ NMR spectroscopy. FTIR spectra (Shimadzu) were recorded using diffused cell technique and $\mathrm{KBr}$ pellets from range 4000 to $400 \mathrm{~cm}^{-1}$. ${ }^{1} \mathrm{H}$ NMR spectra of the co-polymer was recorded in DMSO $\left(\mathrm{d}_{\sigma}\right)$ by using Bruker spectra spin $(400 \mathrm{MHz})$ at $25^{\circ} \mathrm{C}$.

\section{Morphology of CS-g-PNIPAAm}

To study the interior morphology, the sample was fractured by sputter coating with a thin layer of palladium gold alloy and observed under a scanning electron microscope (JEOL JSM5610LV, Tokyo, Japan).

\section{Determination of lower critical solution temperature (LCST)}

The freeze dried nanoparticles were immersed in double distilled water at room temperature and allowed to swell for $24 \mathrm{~h}$ and the LCST was determined by using DSC-60 Differential Scanning Calorimeter (Make - Shimadzu). The thermal analysis was carried out within temperature range 25 to $50^{\circ} \mathrm{C}$ under dry nitrogen atmosphere with a flow rate of $30 \mathrm{~mL} / \mathrm{min}$ and heating rate of $2^{\circ} \mathrm{C} / \mathrm{min}$. Temperatures at the onset point of DSC thermograms were taken as the LCST of the compound.

\section{Preparation of oxaliplatin-loaded CS-g-PNIPAAm nanoparticles by different methods \\ Direct loading method}

The oxaliplatin-loaded chitosan-g-PNIPAAm (OXACS-g-PNIPAAm) nanoparticles were synthesized using similar procedure used for blank nanoparticles (preparation of chitosan-g-PNIPAAm co-polymeric nanoparticles) instead it included an initial addition of oxaliplatin $(100 \mathrm{mg})$ to the solution. After dialysis, the nanoparticles were centrifuged and collected. Dry nanoparticles were obtained by lyophilization and stored at $4^{\circ} \mathrm{C}$. To explore the effect of chitosan content on drug loading efficiency, co-polymeric nanoparticles with variable chitosan content were synthesized and tabulated in Table 1.

\section{Self assembly method}

Five formulations (F1-F5) of oxaliplatin-loaded nanoparticles were prepared by varying drug: polymer ratios using self-assembly method as summarized in Table $2 .^{24}$ The aqueous solutions of specific amount of drug and co-polymer (CP-1) were prepared separately and mixed together. The volume of the mixture was made up to $25 \mathrm{~mL}$ with distilled water. The dispersion was sonicated in ice bath using a prob-type sonicator by varying sonication time such as, $2 \mathrm{~min}$ and $5 \mathrm{~min}$ (Table 2). Dry nanoparticles were obtained by centrifugation and freeze drying.

\section{Determination of particle size and zeta potential}

Particle size of all the formulations was determined by DLS using particle size analyzer (Nanotrac R-150 USA). Samples were prepared by diluting the small quantity of dry nanoparticles in double distilled water with constant stirring for $1 \mathrm{~h}$ and filtered through $0.45 \mu \mathrm{m}$ millipore filter to get the desired nanoparticle dispersion. The mean diameter $( \pm S D)$ was obtained from 6 determinations. The zeta potential was determined by Malvern Zetasizer and the average values of triplicates were taken.

\section{Determination of loading efficiency from direct loading method}

After polymerization, when the nanoparticle suspension was dialyzed for a week, the dialysate has to be ultracentrifuged with $15,000 \mathrm{rpm}$ at $4^{\circ} \mathrm{C}$ for $1 \mathrm{~h}$ and the supernatant is analyzed daily for percent drug content by using HPLC. The mobile phase, Acetonitrile:Acidified water $\mathrm{pH} 3.0(1: 99 \mathrm{v} / \mathrm{v})$, was pumped at a flow rate of $1.4 \mathrm{ml} / \mathrm{min}$ through the column Phenomenex, C18 column, $150 \mathrm{~mm} \times$ $4.6 \mathrm{~mm}, 5 \mu \mathrm{m}$. Oxaliplatin was detected using UV detector at $\lambda_{\max }$ of $210 \mathrm{~nm}$. The standard curve for the quantification of oxaliplatin was linear over the range of $10-60 \mu \mathrm{g} / \mathrm{ml}$ with a correlation coefficient of $0.999 .{ }^{29}$

\section{Determination of loading efficiency from self assembly method}

The percent drug loading efficiency of nanoparticles was analyzed by ultracentrifugation method. The nanoparticle suspension was centrifuged at $75,000 \mathrm{rpm}$ and $4^{\circ} \mathrm{C}$ for $1 \mathrm{~h}$. The supernatant was collected and drug content 
was analyzed by using HPLC. Drug loading efficiency for both the methods was calculated by using the following equation:

$$
\text { Loading efficiency }(\%)=\frac{\text { weight of total drug }- \text { weight of free drug found }}{\text { weight of total drug }} \times 100
$$

\section{Determination of drug content}

The drug content was determined by hydrolyzing the exactly weighed amount of drug loaded nanoparticles $(100 \mathrm{mg})$ in $1 \mathrm{~mol} / \mathrm{L} \mathrm{HCl}$ at $60^{\circ} \mathrm{C}$ for $1 \mathrm{~h}$ till the clear solution was obtained. ${ }^{24}$ Free drug was separated from the nanoparticle by centrifugation at $12000 \mathrm{rpm}$ for 30 min and the drug content was analyzed using HPLC. The data were expressed as the mean value of three independent experiments. F-5

\section{In-vitro drug release study}

Drug loaded nanoparticles $(50 \mathrm{mg}$ ) were resuspended in $10 \mathrm{~mL}$ of phosphate buffer solution of $\mathrm{pH} 7.2$ and the solution was transferred in to 20 eppendorf tubes $(500 \mu l$ each). The tubes were kept in a thermostable water bath at temperature above LCST i.e., $32^{\circ} \mathrm{C}$ for $\mathrm{CP}-2$ and, $35^{\circ} \mathrm{C}$ for $\mathrm{CP}-3$ and $38^{\circ} \mathrm{C}$ for $\mathrm{CP}-4$. The solution was centrifuged at $15,000 \mathrm{rpm}$ for $30 \mathrm{~min}$ and because of the water soluble nature of oxaliplatin, the supernatant was analyzed for drug content by HPLC. By using standard calibration curve, the concentration of drug released was calculated and the percentage drug release was calculated by using the following equation.

$$
\text { DrugRelease }(\%)=\frac{\text { released oxaliplatin from nanoparticles }}{\text { total amount of oxaliplatin in nanoparticles }} \times 100
$$

Drug release study of nanoformulation loaded by self assembly method F-5 was also performed at $25^{\circ} \mathrm{C}$ which was below LCST temperature of the nanoparticles and $\mathrm{pH} 7.5$ as well as at $32^{\circ} \mathrm{C}$ which was above LCST temperature of the nanoparticles and $\mathrm{pH} 7.2$ All release measurements were triplicated for each sample and average values were plotted.

\section{Statistics}

Statistical analysis of the data as described in the experimental sections was performed by one-way analysis of variance (ANOVA) using GraphPad Prism 5.0 software; a value of $\mathrm{P}<0.05$ were regarded to be statistically significance in all cases.

\section{RESULT AND DISCUSSION}

\section{Synthesis of CS-g-PNIPAAm co-polymer}

The co-polymers were successfully synthesized by using NIPAAm and chitosan monomers via surfactant free dispersion copolymerization method with $\mathrm{MBA}$ as a cross linking agent and APS as a radical initiator. Elevated temperature is required for the decomposition of APS to produce sulfate anion radicals and to phase separate the growing PNIPAAm chains to produce colloidal particles. The radicals of APS then interact with the hydroxyl and amino groups of chitosan to form alkoxy radicals which initiate the graft copolymerization of NIPAAm onto CS with crosslinking agent MBA. ${ }^{24,28}$ When APS is used as an initiator in the synthesis of co-polymer, three complex particles were produced such as, PNIPAAm, CS-g-PNIPAAm (negatively charged) and $\mathrm{CS}-\mathrm{NH}_{3}^{+}$(positively charged). These positively charged particles form polyelectrolyte complexes establish electrostatic interactions with excess of chitosan which causes the prevention of the coagulation of particles. ${ }^{30}$

\section{Structure analysis of CS-g-PNIPAAm}

IR spectra of chitosan (A), co-polymer (B) and PNIPAAm $(C)$ were compared in Figure 1. For chitosan, peaks of the $\mathrm{OH}$ stretch appears in the region $3543 \mathrm{~cm}^{-1}$ to $3689 \mathrm{~cm}^{-1}$. CH stretching of heterocyclic ring appears at $2980 \mathrm{~cm}^{-1}$ whereas in the case of pure PNIPAAm it was observed that three characteristic stretching bands of (NH stretching), amide I, and amide II (N-H bending) were present at 3291, 1643, and $1544 \mathrm{~cm}^{-1}$ respectively. For CS-g-PNIPAAm, the several peaks observed in pure chitosan in the region $3543 \mathrm{~cm}^{-1}$ to $3689 \mathrm{~cm}^{-1}$ are diminished in the IR spectrum of synthesized copolymer, which indicates the initiation of linkage between chitosan and NIPAAm at $\mathrm{OH}$ and $\mathrm{NH}$ groups of chitosan. Further the graft polymerization of chitosan and NIPAAm was confirmed by ${ }^{1} \mathrm{H}$ NMR spectroscopy. The ${ }^{1}$ HNMR spectrum of PNIPAAm(Figure2-A) exhibited
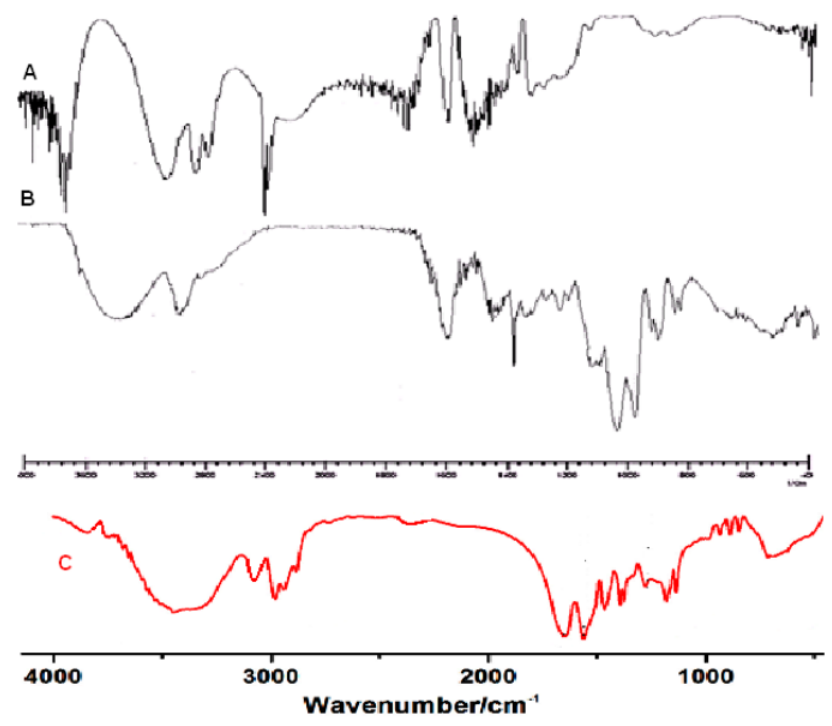

Figure 1: FTIR Spectrum of Chitosan (A), CS-g-PNIPAAm Copolymer (B) and PNIPAAm Homopolymer (C) 


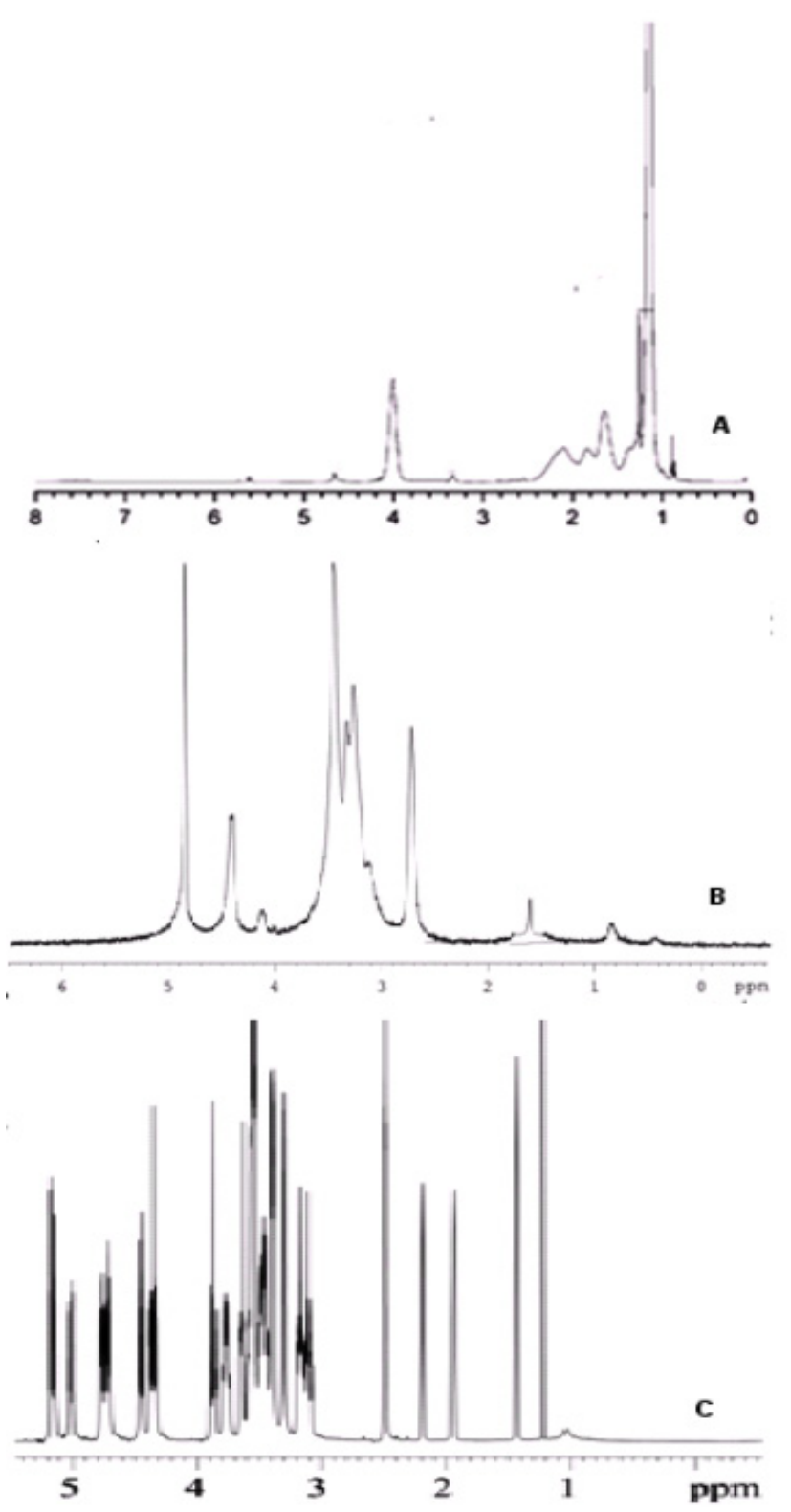

Figure 2: ${ }^{1} \mathrm{H}$ NMR Spectra of PNIPAAm (A), Chitosan (B) and CS-g-PNIPAAm Co-polymer (C).

two peaks $(-\mathrm{CH}-\mathrm{CH} 2)$ at $1.00-2.00 \mathrm{ppm}$, a peak $(-\mathrm{NH}-\mathrm{CH}<)$ at $3.69 \mathrm{ppm}$ and a strong methyl group peak at $1.28 \mathrm{ppm}$. The spectrum of chitosan exhibits characteristic peaks of protons on carbon atoms at $\delta 3.15-3.71 \mathrm{ppm}$, whereas partially acetylated methyl proton peaks appear at $\delta 1.64 \mathrm{ppm}$ and proton on anomeric carbon at $\delta 4.44 \mathrm{ppm}$ (Figure 2-B). But in the spectrum of CS-g-PNIPAAm copolymer (Figure 2-C), signals pertaining to chitosan were a weak peak at $\delta 1.98$ ppm, the peaks of protons on the carbon of chitosan at $\delta 3.10$ - $4.37 \mathrm{ppm}$ and the proton peak of carbon having amine (partially acetamido) groups at $\delta 2.49 \mathrm{ppm}^{30}$ A strong methyl group peak at $\delta 1.23 \mathrm{ppm}$ and two typical peaks $\left(-\mathrm{CH}-\mathrm{CH}_{2}\right)$ at $\delta 1.23-2.20 \mathrm{ppm}$ proved

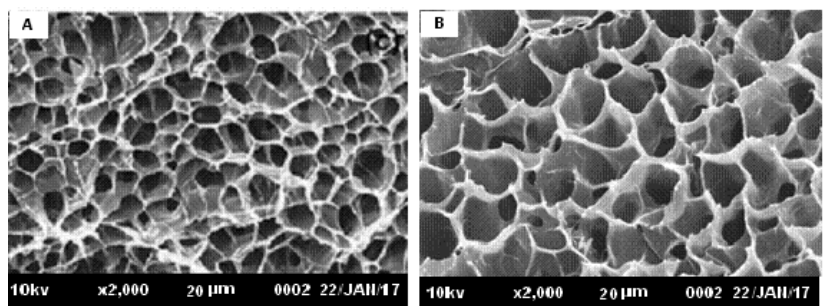

Figure 3: SEM Images of Interior Morphology of PNIPAAm (A) and CS-g-PNIPAAm (B).

the existence of PNIPAAm. From these observations it was confirmed that graft polymerization was successful.

\section{Morphology of CS-g-PNIPAAm co-polymer}

The interior micrographs of PNIPAAm (A) and CSg-PNIPAAm co-polymer (B) are depicted in Figure 3 wherein porous inner structures were observed. Chitosan content in the co-polymer leads to increase in the pore sizes. The pore size of pure PNIPAAm was 4 to $9 \mu \mathrm{g}$ and that of CS-g-PNIPAAm was found to be 8 to $18 \mu \mathrm{g}$. This indicated the incorporation of chitosan has created more pore channels in the co-polymer and highly expanded network for co-polymer has achieved which is a requisite for the swelling/shrinking process of co-polymer.

\section{Effect of chitosan content of co-polymer on drug loading efficiency}

To study the effect of chitosan concentration in co-polymer on drug loading efficiency, three formulations (CP-1,CP-2 and CP-3) of oxaliplatin loaded co-polymeric nanoparticles with varying chitosan concentrations were prepared by direct loading method. Chitosan content, LCST, \% drug loading efficiency ( $\%$ LE), \% drug content ( $\%$ DC), particle size and zeta potential of all the formulations are shown in Table 1 and DSC thermograms of synthesized CS-g-PNIPAAm co-polymeric nanoparticles are shown in Figure 4. The incorporation of hydrophilic co-monomer, chitosan increased the LCST of co-polymer which was a similar finding as that of our previous work. ${ }^{31}$ As the chitosan concentration in co-polymer increased, the $\%$ drug loading efficiency and $\%$ drug content also increases. This could be attributed to the fact that at the temperature above LCST, the increase in weight ratio of chitosan/PNIPAAm would increase the swelling ratio of CS-g-PNIPAAm co-polymer and also, the amount of drug diffused in to the co-polymer which in turn leads to enhance the amount of drug loaded in to the co-polymer. 
Table 1: Preparation of CS-g-PNIPAAm nanoparticles with varying chitosan concentration by direct loading method and their evaluation

\begin{tabular}{|c|c|c|c|c|c|c|c|}
\hline Formulations & $\begin{array}{c}\text { Chitosan } \\
(\mathbf{m g})\end{array}$ & $\begin{array}{c}\text { Oxaliplatin } \\
(\mathbf{m g})\end{array}$ & $\begin{array}{c}\text { LCST } \\
(\mathbf{~} \mathbf{C})\end{array}$ & $\begin{array}{c}\text { Drug Loading } \\
\text { Efficiency } \\
(\%)\end{array}$ & $\begin{array}{c}\text { Drug } \\
\text { Content } \\
(\mathbf{\%})\end{array}$ & $\begin{array}{c}\text { Particle } \\
\text { size } \\
(\mathbf{n m})\end{array}$ & $\begin{array}{c}\text { Zeta } \\
\text { potential } \\
(\mathbf{m V})\end{array}$ \\
\hline CP-1 & 200 & 0.0 & 31.21 & - & - & $238 \pm 22$ & $25 \pm 4.5$ \\
\hline CP-2 & 200 & 100 & 31.44 & $42.81 \pm 4.3$ & $2.9 \pm 0.9$ & $276 \pm 18$ & $27 \pm 6.4$ \\
\hline CP-3 & 250 & 100 & 34.50 & $49.84 \pm 6.1$ & $3.4 \pm 0.7$ & $267 \pm 12$ & $30 \pm 4.3$ \\
\hline CP-4 & 300 & 100 & 37.45 & $55.87 \pm 5.6$ & $4.3 \pm 1.2$ & $254 \pm 16$ & $34 \pm 5.6$ \\
\hline
\end{tabular}

Table 2: Preparation of CS-g-PNIPAAm nanoparticles by self assembly method and their evaluation

\begin{tabular}{|c|c|c|c|c|c|}
\hline \multirow[b]{2}{*}{ Formulations } & \multirow[b]{2}{*}{ Drug:Polymer } & \multicolumn{2}{|c|}{ \% Loading Efficiency } & \multicolumn{2}{|c|}{$\%$ Drug Content } \\
\hline & & Sonication time $2 \mathrm{~min}$ & $\begin{array}{c}\text { Sonication time } \\
5 \mathrm{~min}\end{array}$ & $\begin{array}{c}\text { Sonication time } \\
2 \mathrm{~min}\end{array}$ & $\begin{array}{c}\text { Sonication time } \\
5 \mathrm{~min}\end{array}$ \\
\hline F-1 & $0.5: 10$ & $62.3 \pm 3.4$ & $68.4 \pm 4.5$ & $32.0 \pm 3.3$ & $35.1 \pm 4.4$ \\
\hline $\mathrm{F}-2$ & $1.0: 10$ & $66.7 \pm 4.3$ & $73.8 \pm 3.6$ & $33.8 \pm 2.4$ & $38.7 \pm 4.8$ \\
\hline$F-3$ & $1.5: 10$ & $70.2 \pm 6.8$ & $78 \pm 5.4$ & $35.5 \pm 4.0$ & $41.4 \pm 5.2$ \\
\hline F-4 & $2.0: 10$ & $74 \pm 5.1$ & $81 \pm 4.2$ & $36.2 \pm 2.4$ & $45.0 \pm 6.2$ \\
\hline F-5 & $3.0: 10$ & $77 \pm 6.2$ & $83 \pm 4.4$ & $37.0 \pm 4.3$ & $47.9 \pm 5.4$ \\
\hline
\end{tabular}

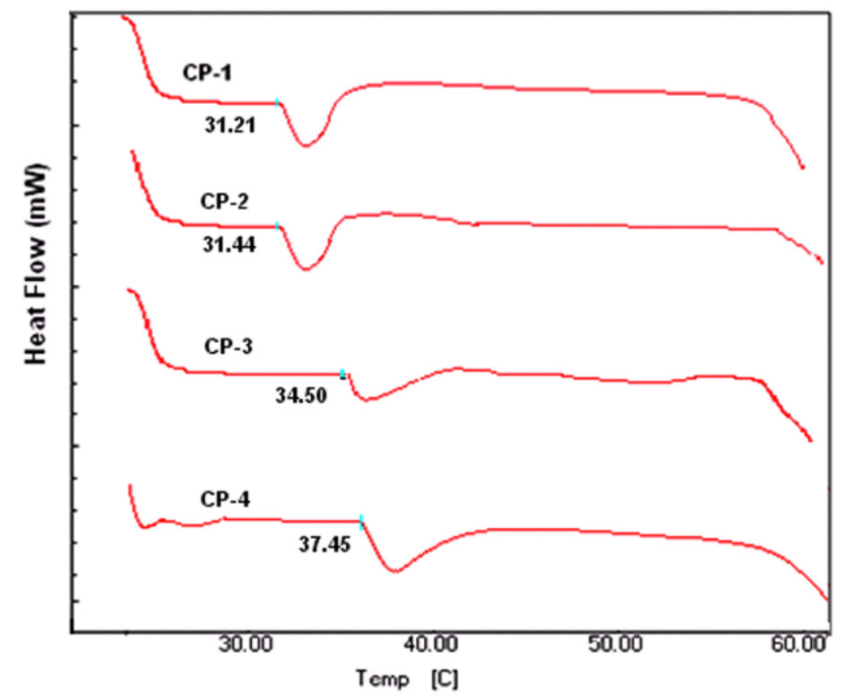

Figure 4: DSC Thermograms of Synthesized CS-g-PNIPAAm Co-Polymeric Nanoparticles

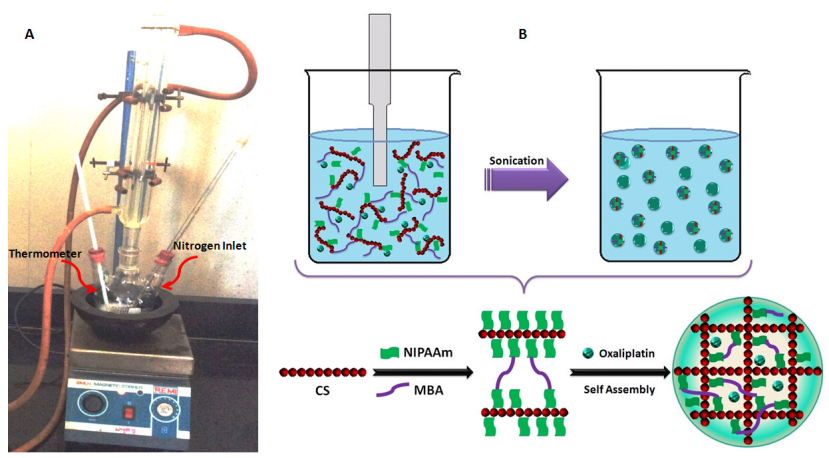

Figure 5: Representation of Drug Loading Methods: A. Direct Loading Method, B. Self Assembly Method

\section{Comparison of drug loading methods for loading efficiency and drug content}

The methods of drug loading are represented in Figure 5. From the obtained \% loading efficiency (\% LE) and drug content $(\%$ DC) for the direct loading method and self-assembly method using sonication (Table 1 and Table 2), it can be inferred that the \% LE and \% DC achieved by self-assembly method was higher than direct loading method. The thermal property (coil to globule transformation above LCST temperature) of grafted PNIPAAm will govern the ability of CS-g-PNIPAAm co-polymer to self-assemble as nanoparticles in aqueous medium. ${ }^{32}$ Due to their self-assembly these nanoparticles could easily encapsulate the slightly water soluble drugs (as shown in Figure 5), thus increasing the \% LE and $\% \mathrm{DC}$ of formulation. In order to achieve the maximum amount of drug incorporated in to nanoparticles, drug: polymer ratio was varied in formulations and it was observed that $\% \mathrm{LE}$ and $\% \mathrm{DC}$ can be increased by increasing the drug: polymer ratio (Table 2 ). The $\% \mathrm{LE}$ and $\% \mathrm{DC}$ were also measured at two different sonication times of 2 min and $5 \mathrm{~min}$ and it was observed that as sonication time increased \% LE and \% DC were also increased.

In direct loading method, the solubility of slightly water soluble drug oxaliplatin is further improved in the presence of acetic acid, which leads to efficient loading of drug in to nanoparticles at above LCST. During dialysis, the unentrapped drug and the drug adsorbed on the surface of nanoparticles was dialysed in to the external solvent. Also the removal of acetic acid was 
always accompanied by the release of oxaliplatin. This is because; the CS-g-PNIPAAm is hydrophilic below LCST, thus most of the entrapped drug gets diffused out of the co-polymeric nanoparticles and removed along with the acetic acid, thereby decreasing the $\% \mathrm{LE}$ and $\%$ DC. The presence of drug in the external solvent was gradually decreased from first to third day and on forth day there was a very little or no drug was found in the external solvent.

\section{Particle size and zeta potential}

The mean particle size obtained for the formulations prepared by direct loading method are shown in Table 1. The particle size of blank nanoparticles was $238 \mathrm{~nm}$ and that of drug loaded nanoparticles ranged from $254 \mathrm{~nm}$ to $276 \mathrm{~nm}$. The increase in particle size of drug loaded nanoparticles is attributed to the drug loading phenomenon. The drug loaded nanoparticles exhibit higher zeta potential than that of blank nanoparticles, indicating the stability of nanoparticles. The particle size and zeta potential of drug loaded nanoparticles (F-5) prepared by self-assembly method were found to be $174 \pm 10 \mathrm{~nm}$ and $48 \pm 11 \mathrm{mV}$, respectively.

\section{Effect of chitosan content of co-polymer on drug release}

The in-vitro drug release profiles of nanoparticles CP-2, CP-3 and CP-4 prepared by direct loading method were depicted in Figure 6. The percent release of drug from CP-2, CP-3 and CP-4 were found to be $52 \%, 61 \%$ and $70 \%$, respectively. From the figure, it can be inferred that, as the chitosan content of co-polymer increased, the $\%$ drug release from nanoparticles at above LCST is increased. The temperature above LCST of co-polymeric nanoparticles was mainly responsible for drug release mechanism from nanoparticles. At above LCST, polymer-polymer interactions are increased and drug polymer interactions are decreased leading to aggregation of nanoparticles (shrinkage) thereby causing the initial burst release of drug which is consistent with all the formulations. This initial burst release follows a swelling controlled release mechanism. At above LCST, the increase in the concentration of chitosan increases the swelling of CS-g-PNIPAAm co-polymer, which facilitates the diffusion controlled release of drug from nanoparticles. Thus, as the chitosan concentration increased, the $\%$ drug release from nanoparticles also increased.

In-vitro drug release of nanoparticles loaded by self assembly method

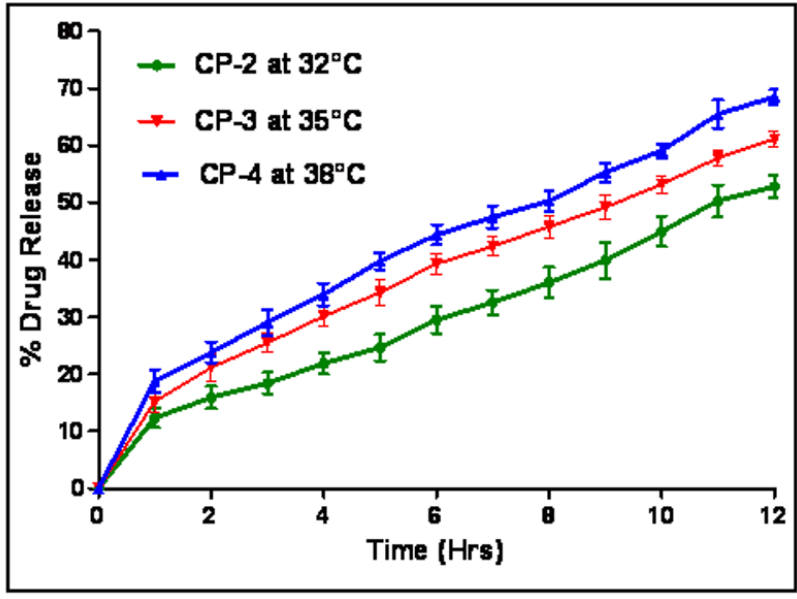

Figure 6: The Drug Release Profile of Oxaliplatin Loaded Nanoparticles Prepared by Direct Loading Method at pH 7.2 and Above LCST of Nanoparticles.

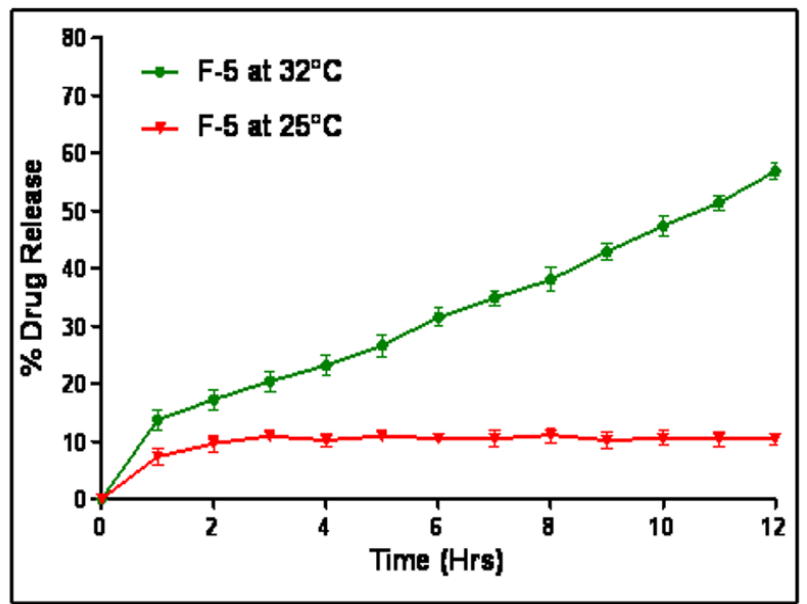

Figure 7: The Drug Release Profile of Oxaliplatin Loaded Nanoparticles Prepared by Self Assembly Method.

Drug release profiles of nanoparticles prepared by self assembly method F-5 at temperatures below $\left(25^{\circ} \mathrm{C}\right)$ and above $\left(32^{\circ} \mathrm{C}\right)$ LCST of nanoparticles with varying $\mathrm{pH}$ were depicted in Figure 7. The drug release at $25^{\circ} \mathrm{C}$ and $\mathrm{pH} 7.5$ was observed about $10 \%$ only, whereas it was increased up to $56 \%$ at $32^{\circ} \mathrm{C}$ and $\mathrm{pH} 7.2$. The drug release was found to be higher at high temperature and less $\mathrm{pH}$ indicating the thermo and $\mathrm{pH}$ responsive property of CS-g-PNIPAAm co-polymer.

\section{CONCLUSION}

CS-g-PNIPAAm co-polymer was successfully synthesized by surfactant free dispersion co-polymerization method using a cross-linking agent MBA and initiator APS. The interior morphology of CS-g-PNIPAAm showed porous inner structure and chitosan content in the co-polymer leads to increase in the pore sizes. The anticancer drug, oxaliplatin was loaded by two methods, direct loading and self assembly method. In direct loading 
method, the drug was loaded during polymerization of co-polymer and by varying chitosan concentration in co-polymer, it was observed that as chitosan content of co-polymer increases, the drug loading and percent drug release at above LCST increases, which was because of the swelling ratio of co-polymer got increased due to the presence of chitosan. In self assembly method, the drug was loaded in co-polymer by using sonicator and it was found that as sonication time and drug:polymer ratio increased, the \% LE and \% DC also increased. In-vitro drug release study revealed the thermo and $\mathrm{pH}$ responsive release of drug from nanoparticles. In conclusion, higher oxaliplatin loading can be achieved by using self assembly method with sonication time $5 \mathrm{~min}$ and drug:polymer ratio of about 3:10 and oxaliplatin release can be controlled by varying chitosan concentration in co-polymer. Future publications in this series will deal with potential application of the optimized CS-g-PNIPAAm co-polymer (for its thermo and $\mathrm{pH}$ responsiveness) for site specific drug delivery.

\section{ACKNOWLEDGEMENT}

Authors are thankful to the KLE University, Belagavi for provinding grant to perform this research work. Authors extend their regards to Dr. Prabhakar Kore Basic Science Research Centre, KLE University Belagavi for providing their amenities to carry out this work. The authors are also thankful to Emcure Pharmaceuticals Limited, Pune, India for providing oxaliplatin pure drug as gift sample.

\section{CONFLICT OF INTEREST}

There are no conflicts of interest.

\section{ABBREVIATION USED}

CS-g-PNIPAAm: chitosan-g-poly(N-isopropylacrylamide); LCST: lower critical solution temperature; LE: loading efficiency; DC: drug content; APS: ammonium persulphate; MBA: N,N-methylenebisacrylamide; NIPAAm: N-isopropylacrylamide; PNIPAAm: poly(N-isopropylacrylamide); HPLC: High performance liquid chromatography; DMSO: dimethylsulphoxide.

\section{REFERENCES}

1. Carreira AS, Gonçalves FAMM, Mendonça PV, Gil MH., Coelho JFJ. Temperature and $\mathrm{pH}$ responsive polymers based on chitosan: Applications and new graft copolymerization strategies based on living radical polymerization. Carbohydrate Polymers 2010;80:618-630.
2. Ru C, Fenghua M, Chao D, Harm-Anton K, Zhiyuan Z. Dual and multistimuli responsive polymeric nanoparticles for programmed site-specific drug delivery. Biomaterials 2013;34: 3647-3657.

3. Mastrotto F, Salmaso S, Alexander C, Mantovani G, Caliceti P. Novel $\mathrm{pH}$-responsive nanovectors for controlled release of ionisable drugs. Journal of Materials Chemistry: B, Materials for Biology and Medicine 2013;1(39):5335-5346.

4. Huang $\mathrm{CH}$, Wang $\mathrm{CH}$, DonTM, Chiu WY. Preparation of $\mathrm{pH}$ - and thermosensitive chitosan PNIPAAm core-shell nanoparticles and evaluation as drug carriers. Cellulose 2013;20:1791-1805.

5. Rejinold NS, Chennazhi KP, Nair SV, Jayakumar R. Thermo-responsive Chitosan-graft-Poly(N-isopropyl acrylamide) co-polymeric nanoparticles for 5 -fluorouracil delivery to breast cancer cells in vitro. J. Nanopharmaceutics Drug Delivery 2013;1(1):18-29.

6. Rejinold NS, Muthunarayanan M, Divyarani W, Sreerekha PR, Chennazhi KP, Nair SV, Tamura H, Jayakumar R. Curcumin-loaded biocompatible thermoresponsive polymeric nanoparticles for cancer drug delivery. J Colloid Interface Sci 2011b;360(1):39-51.

7. Rejinold NS, Sreerekha PR, Chennazhi KP, Nair SV, Jayakumar R. Biocompatible, biodegradable and thermo-sensitive chitosan-g-poly (N-isopropylacrylamide) nanocarriers for curcumin drug delivery. Int J Biol Macromol 2011c; 49(2):161-172.

8. Tian E, Wang J, Zheng Y, Song Y, Jiang L, Zhu D. Colorful humidity sensitive photonic crystal hydrogel Journal of Materials Chemistry 2008;18(10): 1116-1122.

9. Ancla C, Lapeyre V, Gosse I, Catargi B Ravaine V. Designed glucoseresponsive microgels with selective shrinking behavior. Langmuir 2011;27(20):12693-12701.

10. Kanazawa H, Yamamoto K, Matsushima Y, Takai N, Kikuchi A, Sakurai Y, et al. Temperature-responsive chromatography using poly( $N$-isopropylacrylamide)-modified silica. Analytical Chemistry 1996; 68(1):100-105.

11. Maharjan P, Woonton BW, Bennett LE, Smithers GW, De Silva K, Hearn MTW. Novel chromatographic separation - The potential of smart polymers. Innovative Food Science and Emerging Technologies 2008;9(2):232-242.

12. Valmikinathan $\mathrm{CM}$, Chang $\mathrm{W}, \mathrm{Xu}, \mathrm{J}, \mathrm{Yu} \mathrm{X}$. Self assembled temperature responsive surfaces for generation of cell patches for bone tissue engineering Biofabrication 2012; 4(3):035006.

13. Fabienne D, Olivier F, Véronique P. To exploit the tumor micro-environment: Passive and active tumor targeting of nanocarriers for anti-cancer drug delivery. J Cont Rel.2010; 148:135-146.

14. Cui W, Lu X, Cui K, Niu L, Wei Y, and Lu Q. Dual-responsive controlled drug delivery based on ionically assembled nanoparticles. Langmuir 2012; 28,:9413-9420.

15. Soppimath KS, Liu L, Seow WY, Liu S, Powell R, Chan P and Yang YY. Multifunctional core/shell nanoparticles self-assembled from $\mathrm{pH}$-induced thermosensitive polymers for targeted intracellular anticancer drug delivery. Adv. Funct. Mater 2007;17: 355-362.

16. Jong-Ho H, Cheol WC, Hyung-Wook K, Do HK, Tae WK, Hye ML, Cy HK, Chung WC, Young-II J, Dae HK. Dextran-b-poly(L-histidine) copolymer nanoparticles for $\mathrm{pH}$-responsive drug delivery to tumor cells. International Journal of Nanomedicine 2013;8: 3197-3207.

17. Chen C, Kim TH, Wu WC, Huang C, Wei H, Mount CW, Tian Y, Jang S, Pun $\mathrm{SH}$, Jen $\mathrm{K}$. pH-dependent, thermosensitive polymeric nanocarriers for drug delivery to solid tumors. Biomaterials 2013;34:4501-4509.

18. Hu X, Hao X, Wu Y, Zhang J, Zhang X, Wang PC, Zou G and Liang X. Multifunctional hybrid silica nanoparticles for controlled doxorubicin loading and release with thermal and $\mathrm{pH}$ dual response. J. Mater. Chem. B 2013;1:1109-1118.

19. Rejinold NS, Sreerekha PR, Chennazhi KP, Nair SV, Jayakumar R. Biocompatible, biodegradable and thermo-sensitive chitosan-g-poly (N-isopropylacrylamide) nanocarriers for curcumin drug delivery. Int $\mathrm{J}$ BiolMacromol.2011;49(2):161-172.

20. Chen C, Mingzhu L, Chunmei G, Shaoyu L, Jiucun C, Xiyong Y, Enyong D, Chuanming Y, Jing G, Guijia C. A convenient way to synthesize comb-shaped chitosan-graft-poly(N-isopropylacrylamide) copolymer. Carbohydr Polym 2013; 92: 621- 628.

21. Ward MA and Georgiou TK. Thermoresponsive polymers for biomedical applications. Polymers 2011;3:1215-1242. 
Li F, Wu H, Zhang H, Li F, Yang TH, Gu CH, et al. Novel super pH sensitive nanoparticles responsive to tumor extracellular $\mathrm{pH}$. Carbohydr Polym 2008;73(3):390-400

23. Li F, Wu H, Zhang $\mathrm{H}, \mathrm{Gu} \mathrm{CH}$, Yang Q. Antitumor drug paclitaxel loaded $\mathrm{pH}$-sensitive nanoparticles targeting tumor extracellular $\mathrm{pH}$. Carbohydrate Polymers.2009;77(4): 773-778.

24. Cunxian D, Dianrui Z, Feihu W, Dandan Z, Lejiao J, Feifei F, et al. Chitosang-poly(N-isopropylacrylamide) based nanogels for tumor extracellular targeting. Int J Pharm. 2011; 409: 252-259.

25. Zhang T, Li G, Guo L, Chen H. Synthesis of thermo-sensitive CS-g-PNIPAM/ CMC complex nanoparticles for controlled release of 5-FU. International Journal of Biological Macromolecules 2012;51:1109-1115.

26. Chih-Hao H, Cheng-Fan W,Trong-Ming D, Wen-Yen C. Preparation of $\mathrm{pH}$ - and thermosensitive chitosan-PNIPAAm core-shell nanoparticles and evaluation as drug carriers. Cellulose 2013;20:1791-1805.

27. Jocic D, Tourrette A, Glampedaki P, Warmoeskerken MMCG. Application of temperature and $\mathrm{pH}$ responsive micro hydrogels for functional finishing of cotton fabric. Materials Technology 2009;24(1):14-23.

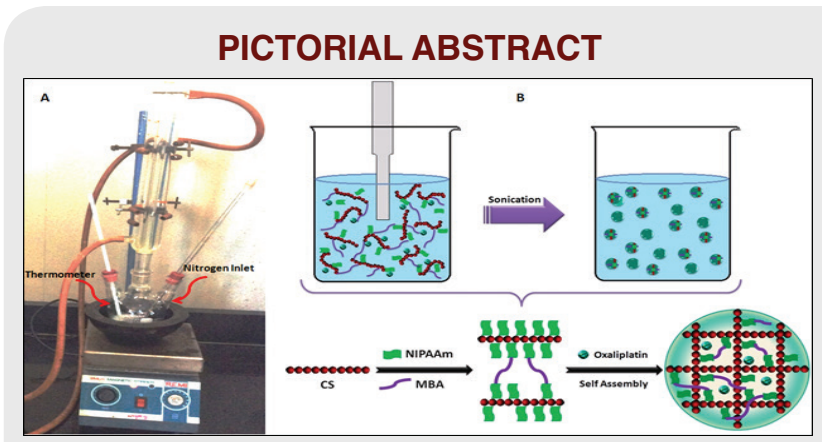

\section{About Authors}

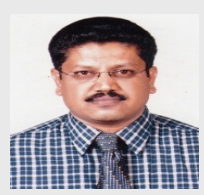

Anand Panchakshari Gadad: Is a Professor and Head, Department of Pharmaceutics, KLE University's College of Pharmacy, K.L.E University, Belagavi $\mathrm{He}$ is working on areas of targeted drug delivery system viz., Gastroretentive drug delivery system, Polymeric nanoparticles, enhancing solubility of poorly soluble drugs, etc.

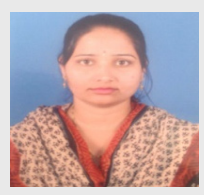

Archana Sidagouda. Patil: Is an Assistan Professor, Department of pharmaceutics, KLE University's College of Pharmacy, K.L.E University, Belagavi. She is working in the area of targeted drug delivery systems viz, $\mathrm{pH}$ and temperature responsive co-polymeric nanoparticles, pulsatile drug delivery systems as well as synthesis and characterization of graft co-polymers for intelligent drug delivery etc.
28. Lee CF, W en CJ, Chiu WY. Synthesis of poly(chitosan-N-isopropylacrylamide) complex particles with the method of soapless dispersion polymerization. $J$ PolymSci Part A: Polym Chem 2003;4:2053-2063.

29. The United States Pharmacopeial Convention. Revision Bulletin Oxaliplatin Official October 1, 2012

30. Chiu YL, Chen SC, Su CJ, Hsiao CW, Chen YM, Chen HL, Sung HW. $\mathrm{pH}$-triggered injectable hydrogels prepared from aqueous $\mathrm{N}$-palmitoyl chitosan: in vitro characteristics and in vivo biocompatibility. Biomaterials 2009;30:4877-4888

31. Patil AS, Gadad AP, Hiremath RD and Dandagi PM. Exploration of the effect of chitosan and crosslinking agent concentration on the properties of dual responsive chitosan-g-poly ( $\mathrm{N}$-isopropylacrylamide) co-polymeric particles. J Polym Environ 2017; doi:10.1007/s10924-017-0971-z

32. Sosnika A, Imperialeb JC, Vázquez-Gonzálezd B, Raskina MM, MunozMunoze F, Burillod G, Cedillod G, Bucio E. Mucoadhesive thermoresponsivechitosan-g-poly( $\mathrm{N}$-isopropylacrylamide) polymeric micelles via a one-pot gamma-radiation-assisted pathway. Colloids and Surfaces B: Biointerfaces 2015;136:900-907.

\section{SUMMARY}

- CS-g-PNIPAAm co-polymer was successfully synthesized by surfactant free dispersion co-polymerization method and graft co-polymerization was confirmed by FTIR and ${ }^{1} \mathrm{H}$ NMR spectras.

- Two different drug loading methods like direct drug loading during polymerization and self-assembly methods were studied for oxaliplatin loading. The drug loaded nanoparticles were evaluated for particle size, zeta potential, percent drug loading efficiency, percent drug content and in-vitro drug release study.

- In direct loading method, as the concentration of chitosan in co-polymer increases, LCST, the \% drug loading efficiency and \% drug content also increases.

- The nanoparticles obtained by self assembly method showed higher \% LE and \% DC as compared to direct loading method. Due to self-assembly of nanoparticles these could easily encapsulate the slightly water soluble drugs, thus increasing the \% LE and \% DC of formulation. Also as the drug: polymer ratio and sonication time increased the \% LE and \% DC increased.

- The in-vitro drug release profiles of nanoparticles CP-2, CP-3 and CP-4 prepared by direct loading method were found to be $52 \%, 61 \%$ and $70 \%$, respectively. This indicates that as chitosan content of co-polymer increased, the \% drug release from nanoparticles at above LCST was increased.

- Nanoparticles prepared by self assembly method (F-5) showed the maximum drug loading and drug release at above LCST ( $\mathrm{pH} 7.2)$ and trace amount of drug release below LCST ( $\mathrm{pH} 7.5$ ), indicating thermo and $\mathrm{pH}$ responsive delivery of oxaliplatin.

Cite this article: Patil AS, Gadad AP. Development and Evaluation of High Oxaliplatin Loaded CS-g-PNIPAAm CoPolymeric Nanoparticles for Thermo and $\mathrm{pH}$ Responsive Delivery. Indian Journal of Pharmaceutical Education and Research. 2018;52(1):62-70. 